\title{
Effect of natural ventilation on the boundary layer separation and near-wake vortex shedding characteristics of a sphere
}

\author{
G. K. Suryanarayana, A. Prabhu
}

\begin{abstract}
Experiments were conducted in water and wind tunnels on spheres in the Reynolds number range $6 \times 10^{3}$ to $6.5 \times 10^{5}$ to study the effect of natural ventilation on the boundary layer separation and near-wake vortex shedding characteristics. In the subcritical range of $\operatorname{Re}\left(<2 \times 10^{5}\right)$, ventilation caused a marginal downstream shift in the location of laminar boundary layer separation; there was only a small change in the vortex shedding frequency. In the supercritical range $\left(R e>4 \times 10^{5}\right)$, ventilation caused a downstream shift in the mean locations of boundary layer separation and reattachment; these lines showed significant axisymmetry in the presence of venting. No distinct vortex shedding frequency was found. Instead, a dramatic reduction occurred in the wake unsteadiness at all frequencies. The reduction of wake unsteadiness is consistent with the reduction in total drag already reported. Based on the present results and those reported earlier, the effects of natural ventilation on the flow past a sphere can be categorized in two broad regimes, viz., weak and strong interaction regimes. In the weak interaction regime (subcritical $R e$ ), the broad features of the basic sphere are largely unaltered despite the large addition of mass in the near wake. Strong interaction is promoted by the closer proximity of the inner and outer shear layers at supercritical Re. This results in a modified and steady near-wake flow, characterized by reduced unsteadiness and small drag.
\end{abstract}

\section{1}

\section{Introduction}

Several methods have been used in the past to reduce the drag of 2-D and 3-D bluff bodies. Reviews by Zdravkovich

Received: 8 September 1998 /Accepted: 1 January 2000

G. K. Suryanarayana $(\varangle)$

National Trisonic Aerodynamic Facilities

National Aerospace Laboratories, Bangalore, India

\section{A. Prabhu}

Department of Aerospace Engineering

Indian Institute of Science, Bangalore, India

The authors wish to thank Professor Dr. G.E.A. Meier and Dr. F.R. Grosche, DLR Institute for Experimental Fluid Mechanics, Göttingen, Germany, for their support in conducting the water tunnel experiments. Useful discussions with Dr. P.R. Viswanath, Experimental Aerodynamics Division, National Aerospace Laboratories, Bangalore, India, are acknowledged. The authors also thank the German Academic Exchange Service (DAAD), Bonn and New Delhi for the financial support for the work carried out in Germany.
(1981) and Viswanath (1994) give a wide coverage of the various passive techniques and their limitations in the context of 2-D and axisymmetric bases in different speed regimes. In contrast to $2-\mathrm{D}$ bodies, very little is understood on the general features of the near-wake structure of 3-D bodies except that vortex shedding is less intense, as discussed in Mair and Maull (1971). In earlier studies by Suryanarayana et al. (1993) and Suryanarayana and Meier (1995), attempts were made to understand some of the major aspects of the near-wake structure of a sphere (which represents an idealized 3-D bluff body) and to control the same for drag reduction using natural/passive ventilation. Natural ventilation of a sphere involves interconnecting the stagnation zone and the base through an internal cylindrical duct. As a result of the pressure difference, a natural bleed occurs into the near wake. Aspects of drag reduction, flow-field visualization and surface pressure distributions with and without natural ventilation have already been published. As reported therein, ventilation causes a large reduction $(65 \%)$ in total drag for supercritical Reynolds numbers $\left(>4 \times 10^{5}\right)$ due to strong interaction between the separated external flow and the vent flow, promoted by their close proximity. The reduction in drag was shown to be caused by substantial base pressure recovery. At subcritical $\operatorname{Re}\left(<2 \times 10^{5}\right)$, there was a marginal change in the total drag. Visualizations of the near wake in the supercritical Reynolds number ( $R e$, based on diameter $D$ ) regime indicated that ventilation leads to modification/suppression of the randomly rotating vortex structure, which is known to be associated with the unvented sphere.

As shown by the early experiments of Wieselberger and Prandtl (1914), downstream shift of the location of boundary layer separation on a sphere causes a drag reduction. When the location is inferred from a finite number of pressure ports in an experiment, the result is an incomplete description, especially at supercritical Re when the separation is non-axisymmetric. A global visualization of the external flow is more useful in such situations.

In the case of a circular cylinder, Roshko (1961) has shown that the total drag and Strouhal number of vortex shedding are closely related. Such a comparison for a sphere shows only a weak correlation. This leads one to speculate that vortex shedding, even at subcritical $R e$, may not be a very important feature of the flow past a sphere as far as the mean-flow dynamics in the near wake is concerned. Such a suggestion has been made by Viswanath (1994) in the context of low-speed axisymmetric base flow at relatively high $R e$. In 2-D bluff-body flows, any passive or even energetic technique used for drag reduction leads 
to an increase in the vortex shedding frequency. Typical examples are the splitter-plate experiments of Roshko (1954), the vented circular cylinder experiments of Igarashi (1978) and base-bleed experiments of Bearman (1967).

In the light of the above, the present experiments were undertaken to study the gross features of boundary layer separation and reattachment as well as near-wake vortex shedding for the flow past a sphere with and without ventilation. The focus of the study is the effect of venting at supercritical $R e$ when ventilation reduces the total drag by $65 \%$ of the basic value. Hence, the identical sphere model which was used in drag measurements (Suryanarayana et al. 1993) was used for the present investigations also. In this paper, the results on the basic or unvented sphere are first reported along with the data available in the literature on spheres. The changes caused by natural ventilation are discussed separately.

\section{2}

\section{Experiments}

Experiments were conducted in a water tunnel (test section size $0.25 \mathrm{~m} \times 0.33 \mathrm{~m}$ ) at the DLR Institute for Experimental Fluid Mechanics, Göttingen, Germany, and in a wind tunnel (test section size $1.5 \mathrm{~m} \times 1.5 \mathrm{~m}$ ) at the $\mathrm{Na}$ tional Aerospace Laboratories (NAL), Bangalore, India, covering a wide range of $R e$ from $6.0 \times 10^{3}$ to $6.5 \times 10^{5}$. The free-stream turbulence intensities in the tunnels are about $0.5 \%$ and $0.12 \%$, respectively. A vertically supported wooden sphere $79 \mathrm{~mm}$ in diameter, as shown in Fig. 1a, was used in the water tunnel at DLR in the range $6.0 \times 10^{3}<R e<2.0 \times 10^{4}$. A 200 -mm-diameter Styrofoam sphere was used in the wind tunnel at NAL in the range $6.0 \times 10^{4}<R e<6.5 \times 10^{5}$. The sphere was held asymmetrically, as shown in Fig. $1 \mathrm{~b}$, using a sting $10 \mathrm{~mm}$ in diameter. Vent holes of diameter $\left(D_{\text {vent }}\right) 12 \mathrm{~mm}$ and $30 \mathrm{~mm}$ were drilled on the respective spheres across the stagnation region to the base region to provide a vent area ratio $A_{\mathrm{v}}$ of $2.25 \%\left(=D_{\text {vent }}^{2} / D^{2}\right)$. Figure $1 \mathrm{c}$ shows a photograph of the model mounted in the test section of the wind tunnel at NAL. A detailed discussion on support interference effects, justifying the use of an asymmetric support in the wind tunnel is given in Suryanarayana (1996).

Surface-flow visualization experiments were carried out to estimate the mean locations of boundary layer separation and reattachment. A mixture of titanium dioxide, oleic acid, and oil was used for this purpose. A hot-film probe in the water tunnel and a hot-wire probe in the wind tunnel were used to measure the $u^{\prime}$ fluctuations in the near wake. The probe was located at several locations in the near wake of the sphere models.

3

\section{Results and discussion}

3.1

\section{Boundary layer separation characteristics - unvented sphere}

Figures $2 \mathrm{a}$ and $\mathrm{b}$ show typical surface-flow patterns oblained at $\operatorname{Re}=6.5 \times 10^{4}$ and $6.5 \times 10^{5}$ in the wind tunnel. a
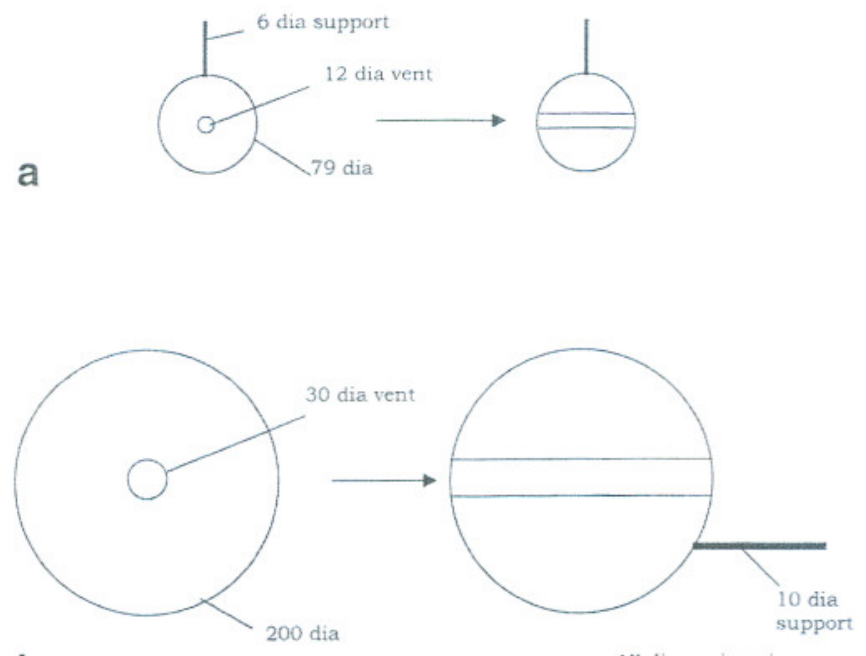

$58:$

b

All dimensions in $\mathrm{mm}$

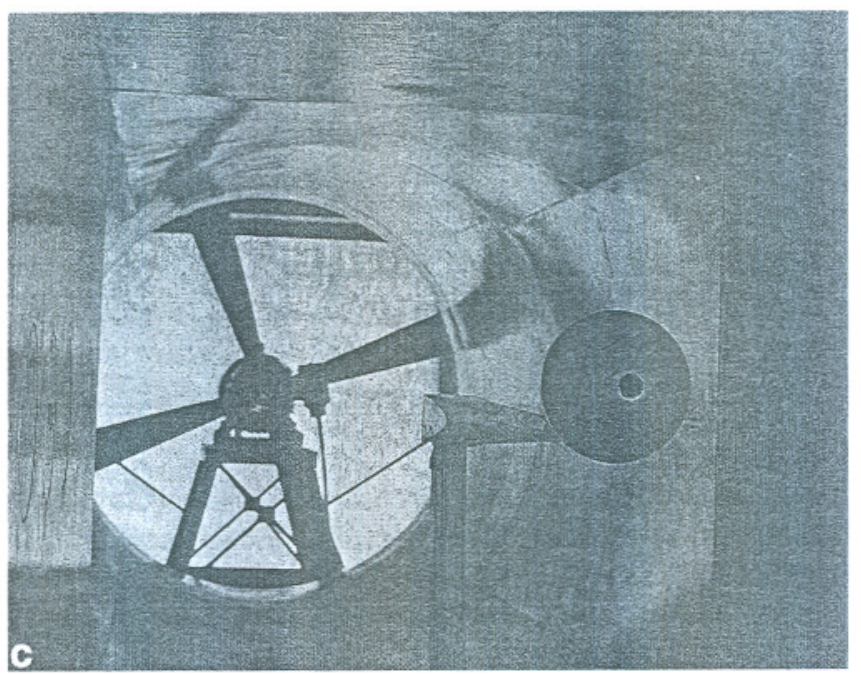

Fig. 1a-c. Details of the sphere models used in the experiments: a sphere model used in water tunnel tests; b sphere model used in wind tunnel tests; $c$ photograph of the sphere mounted in the $1.5-\mathrm{m}$ wind tunnel at NAL

In the subcritical regime (Fig. 2a), axisymmetric laminar separation at $\theta_{\mathrm{ls}} \approx 80^{\circ}$ is indicated. At a much lower Re of 1700 (experiments in water tunnel), separation occurred at $\theta_{1 \mathrm{~s}} \approx 90^{\circ}$ (Suryanarayana et al. 1993). The upstream shift of $\theta_{1 \mathrm{~s}}$ in the range $1.7 \times 10^{3} \leq R e \leq 6.5 \times 10^{4}$ may be related to the shear layer transition location, as found by Achenbach (1972). In the supercritical regime (Fig. 2b), laminar separation at $\theta_{1 \mathrm{~s}} \approx 105^{\circ}$, transitional/turbulent bubble reattachment at $\theta_{\mathrm{br}} \approx 115^{\circ}$ and turbulent separation at $\theta_{\mathrm{ts}} \approx 155^{\circ}$ are shown. It may be noted that, in the supercritical regime, three-dimensionality of the separation and reattachment lines (skewed with respect to the streamwise axis) are evident, whereas the separation line is generally axisymmetric in the subcritical regime. Such a three-dimensionality has also been reported by Taneda (1978).

Figure 3 shows the variation in the location of laminar boundary layer separation as a function of $R e$ from the present experiments. Also shown are the results based on visual observations reported by Raithby and Eckert (1968), Taneda (1978) and Arakeri and Ram (1984). The locations 


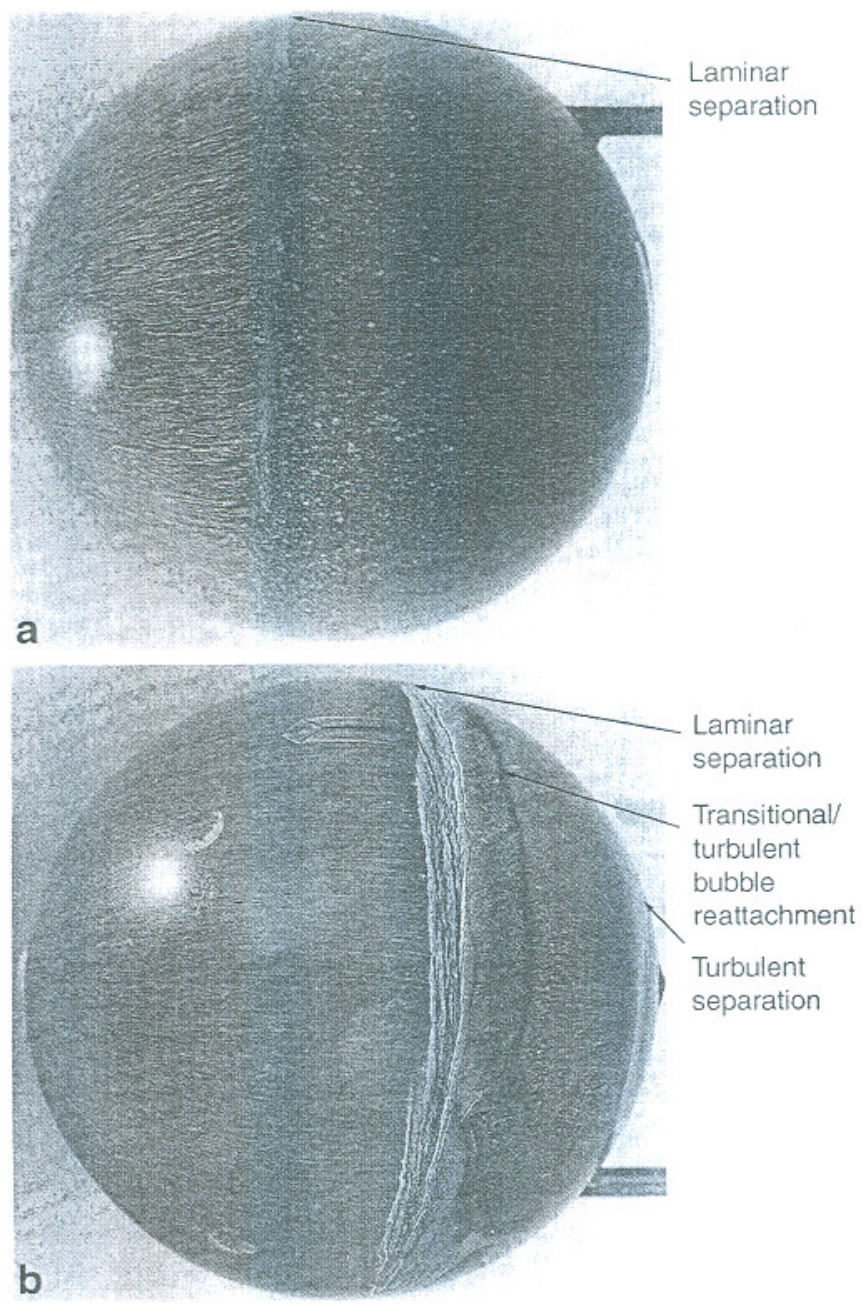

Fig. 2a, b. Surface-flow patterns on the unvented sphere: a $R e=6.5 \times 10^{4} ; \mathrm{b} R e=6.5 \times 10^{5}$

determined through skin-friction measurements reported by Achenbach (1972) and surface pressure measurements by Fage (1936) are also included. Typical uncertainty bands, as mentioned by the individual authors, are also marked. As may be expected, a certain degree of uncer- tainty is inherent in inferring the separation and reattachment lines in each technique employed. For example, the locations inferred from surface-flow patterns (as done now) could have an error of $\pm 2-3 \mathrm{~mm}$, which would translate into a corresponding percentage error in the location depending on the sphere diameter. The uncertainty band at higher $R e$ is inherently high because of skewness. The minimum and maximum variation (as much as $10^{\circ}$ ) from the mean is considered as the uncertainty band, as also done by Taneda (1978). Dissimilarities in the supports used, their interference on the flow and different freestream turbulence intensities in different sets of experiments also contribute to scatter in the data.

It is evident from Fig. 3 that excellent agreement exists among the different sets of data in the subcritical regime, including the curve fit $\left[\theta=78+275 R e^{-0.37}\right]$ recom mended by Clift et al. (1978). As the critical regime is approached, the separation location rapidly shifts downstream; the limited data available in the literature suggest that the laminar separation location may settle around $100^{\circ}$ to $110^{\circ}$ towards the end of the Reynolds number range.

Figure 4 shows the variation in the transitional/turbulent bubble reattachment position with $R e$. The results clearly show that the separated shear layer does not reattach on the sphere (and hence no separation bubble) for $R e<2 \times 10^{5}$ (the shear layer closure occurs in the near wake). As $R e$ is increased, the bubble reattachment position also shifts downstream in the critical regime, in accordance with the movement of $\theta_{\mathrm{ls}}$, and seems to settle down to a value around $115^{\circ}$ at supercritical Re. The present data clearly show that the reattachment location moves upstream (by a few degrees) before moving downstream in the critical regime.

The location of turbulent boundary layer separation is shown plotted in Fig. 5 as a function of Re. The present results show a gradual downstream shift of $\theta_{\mathrm{ts}}$ with $R e$, similar to those of $\theta_{\mathrm{ls}}$ and $\theta_{\mathrm{br}}$, and appears to settle around a value of $\theta_{1 \mathrm{~s}} \approx 155^{\circ}$. On the other hand, the data of Achenbach (1972) exhibit a nearly constant value (around $120^{\circ}$ ), which may be due to the high free-stream turbulence intensity in his $(0.45 \%)$ as compared with the present $(0.12 \%)$ experiments; also, this value may represent the

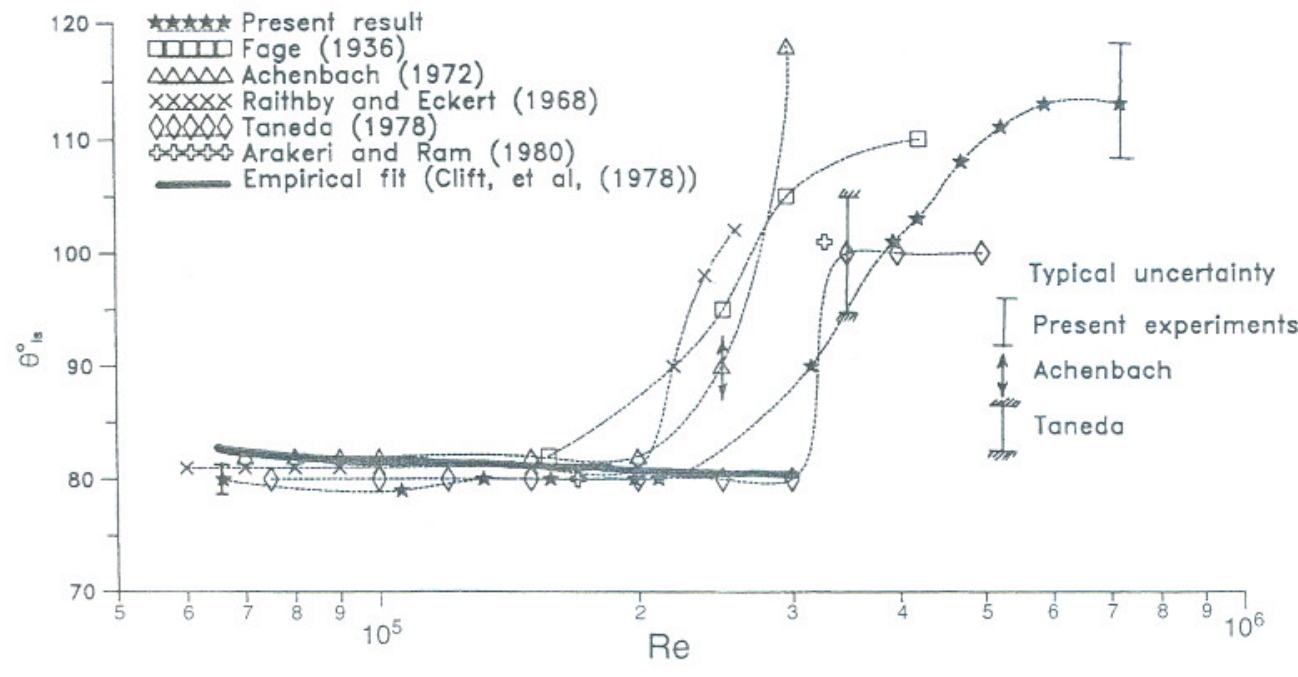

Fig. 3. Comparison of the location of laminar boundary layer separation on unvented sphere with data in the literature 


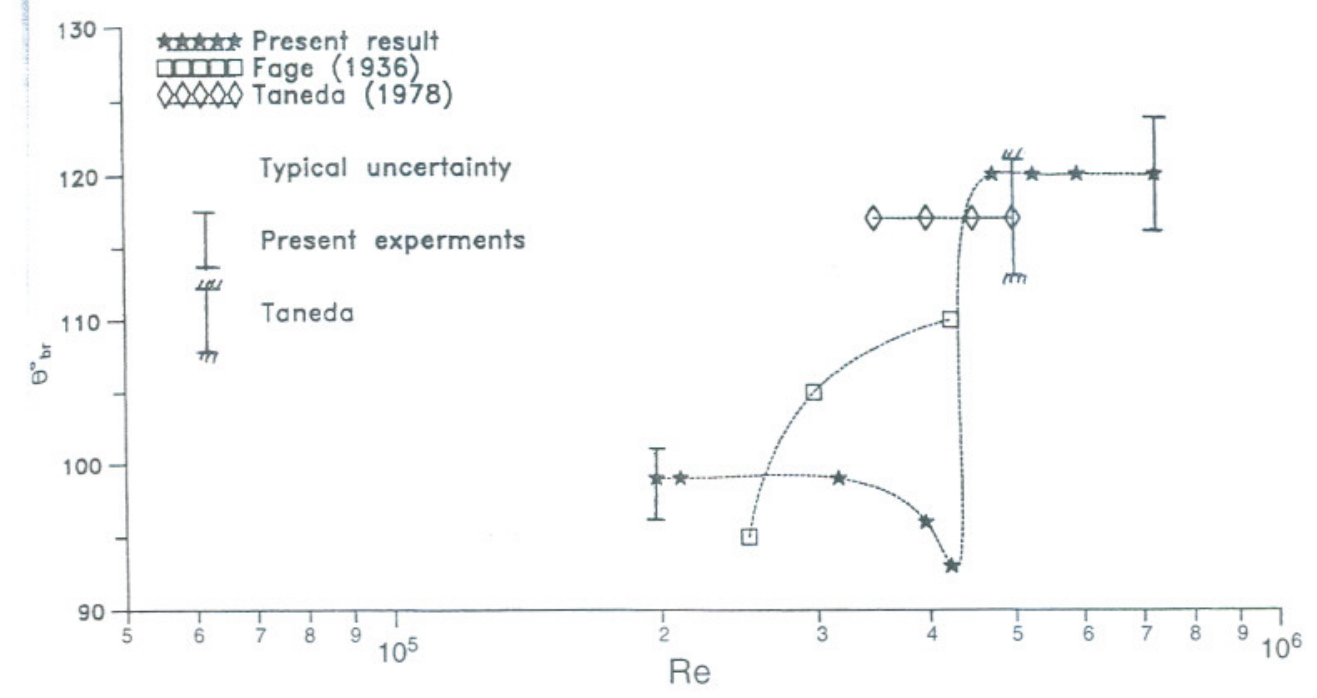

Fig. 4. Comparison of the location of bubble reattachment on unvented sphere with data in the literature

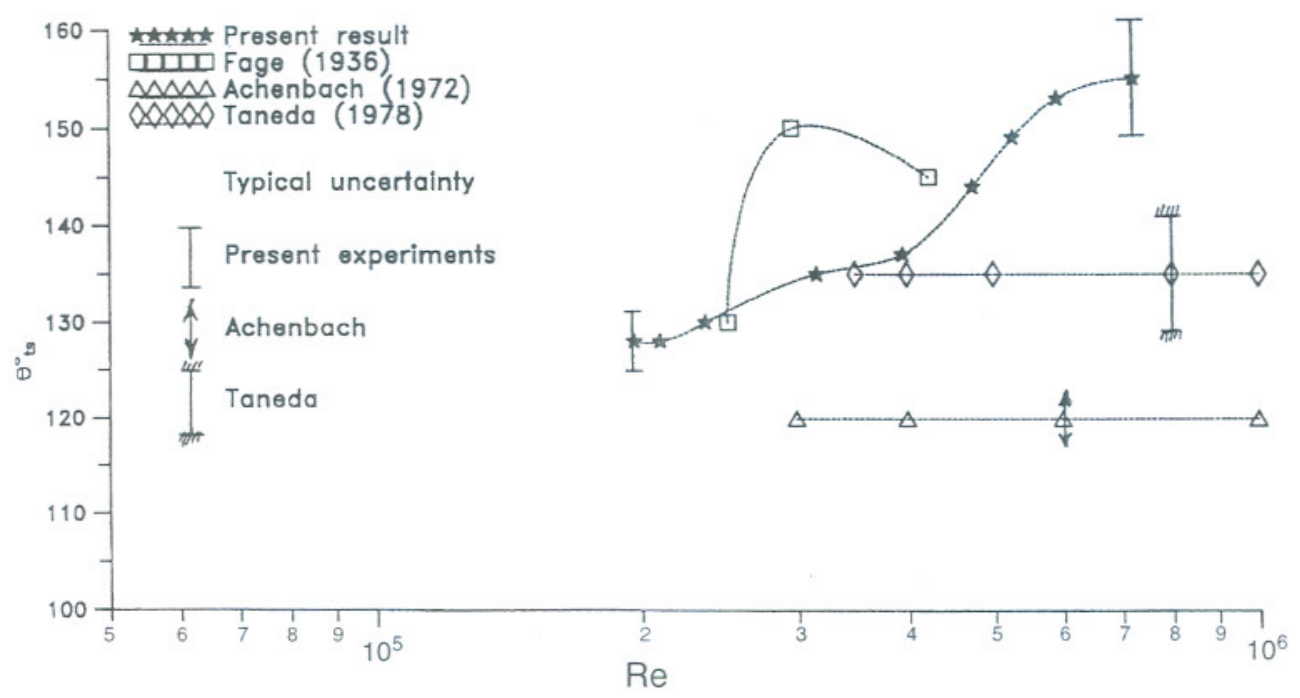

Fig. 5. Comparison of the location of turbulent boundary layer separation on unvented sphere with data in the literature asymptotic value of $\theta_{\mathrm{ts}}$, which is to be expected at much higher $R e$, with transition occurring in the boundary layer on the sphere rather than in the shear layer following the aminar separation.

3.2

\section{lortex shedding - unvented sphere}

Typical power spectra of $u$ ' fluctuations obtained in the water and wind tunnels are shown in Figs. 6 and 7, reipectively. These results clearly show (Figs. 6a-7d) the xistence of vortex shedding in the range $6.4 \times 10^{3} \leq$ $R e \leq 2.6 \times 10^{5}$. It may be noted that the second peak at $1.9 \mathrm{~Hz}$ seen in Fig. $6 \mathrm{a}-\mathrm{c}$ at $R e=6.4 \times 10^{3}$ to $9.6 \times 10^{3}$ is not the high-frequency mode that was observed by Kim and Durbin (1988) and Sakamoto and Haniu (1990), since his peak was found to be associated with some electrical roise in the measurement system. In essence, the highrequency mode which is known to exist in the Re range up to 8000 (discussed in the above references) was not bserved in the present experiments even at $R e \approx 6000$, robably due to the relatively higher freestream turbuence intensity $(\approx 0.5 \%)$ in the present experiments. In the critical and supercritical $R e$ regimes, no distinct peak frequency is observed, as shown in Fig. 7e at $R e=6.5 \times 10^{5}$. An absence of vortex shedding for $R e>3 \times 10^{5}$ has also been reported by Cometta (1957) and Achenbach (1974) through hot-wire measurements and Taneda (1978) by visual observations of smoke-flow patterns. The results of Strouhal number $\left(\mathrm{S}_{\mathrm{t}}=f_{\mathrm{s}} D / U_{\infty}\right)$ obtained from these experiments, as shown in Fig. 8, indicate excellent consistency of the present data with the published data.

\section{3}

\section{Effect of ventilation on the boundary layer separation} characteristics

Figure 9 shows the effect of ventilation at a typical subcritical Re of $6.5 \times 10^{4}$. It may be seen that $\theta_{1 \mathrm{~s}}$ is delayed by $2-3^{\circ}$, with the shear layer closure occurring in the near wake in both the cases. Thus, no separation bubble is seen in the subcritical regime, with or without ventilation. The changes in the surface-flow patterns are consistent with the surface pressure measurements reported by Suryanarayana et al. (1995). 

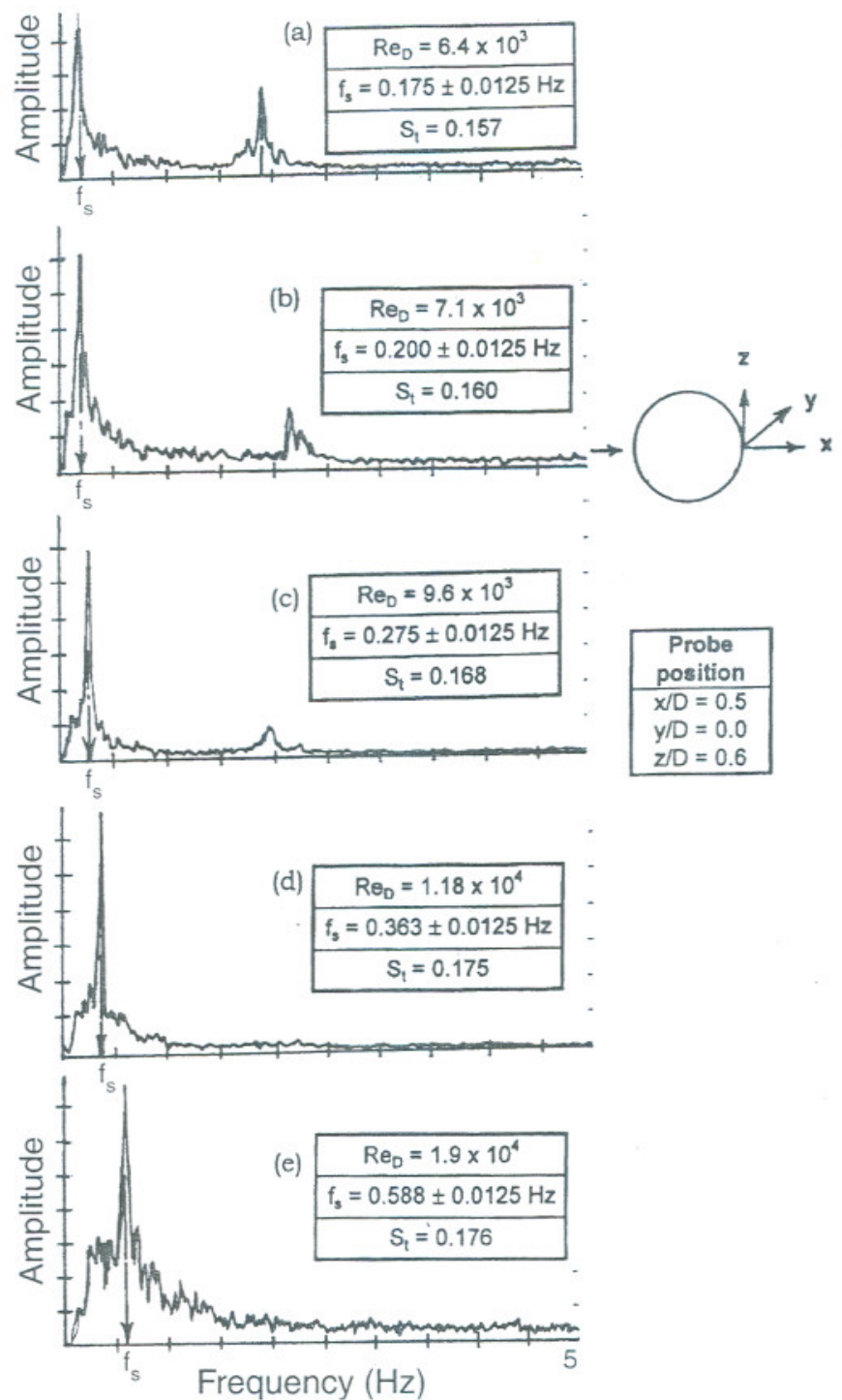

Fig. 6. Typical power spectra of $u^{\prime}$ fluctuations behind the unvented sphere in water tunnel

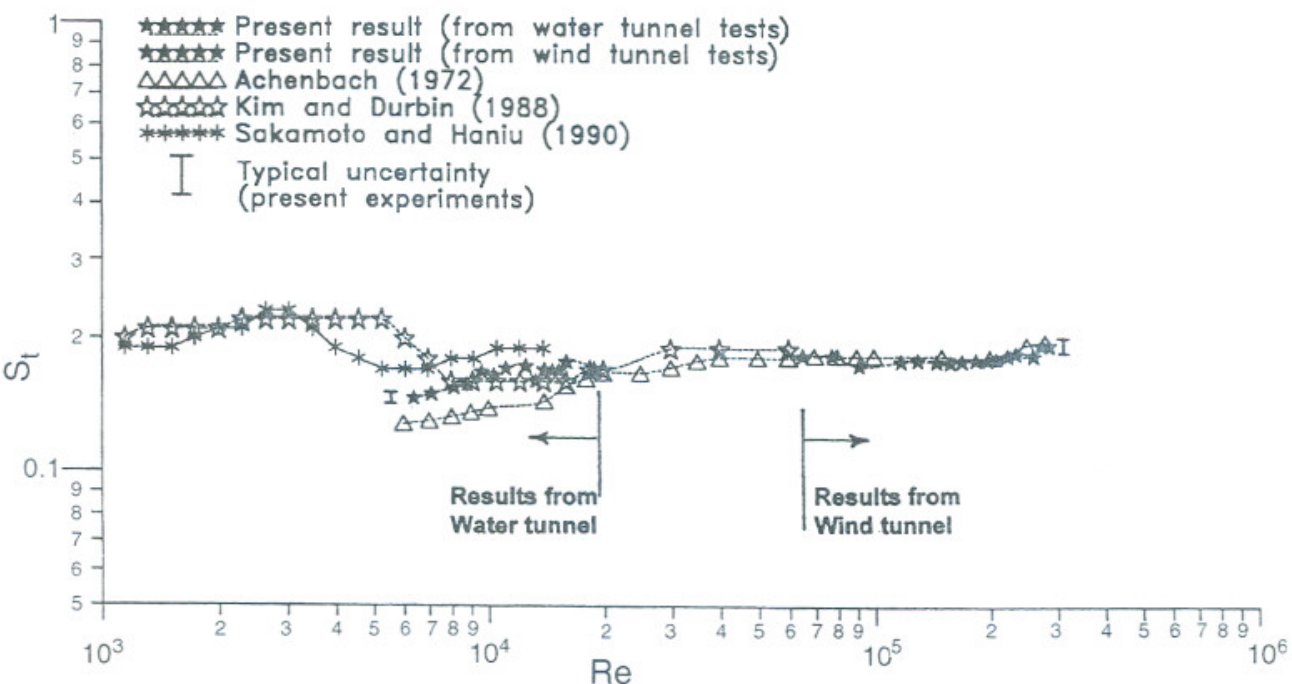

Fig. 8. Comparison of Strouhal number of unvented sphere with data in the literature

Fig. 7. Typical power spectra of $u^{\prime}$ fluctuations behind the unvented sphere in wind tunnel

As indicated in Fig. 10, the surface-flow features at supercritical Re exhibit greater axisymmetry and a downstream shift $\left(\approx 5^{\circ}\right)$ in the locations of $\theta_{\mathrm{ls}}, \theta_{\mathrm{br}}$, and $\theta_{\mathrm{ts}}$. The improved symmetry of the flow is associated with the stabilization of the 3-D randomly rotating horseshoe vortical la structure by natural ventilation, as observed on a laser light sheet by Suryanarayana et al. (1993). The effects of venti- 

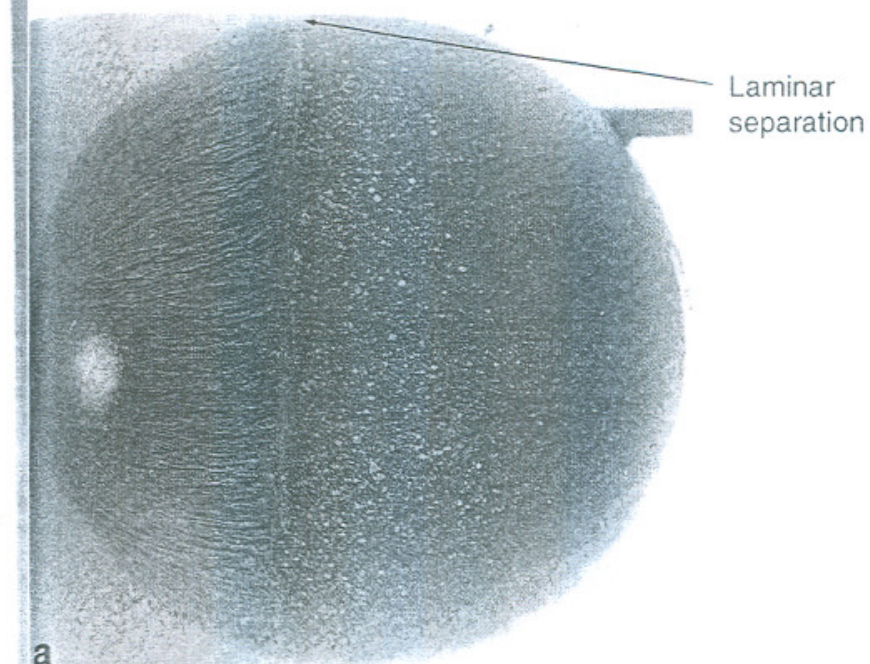

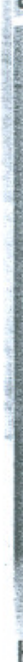
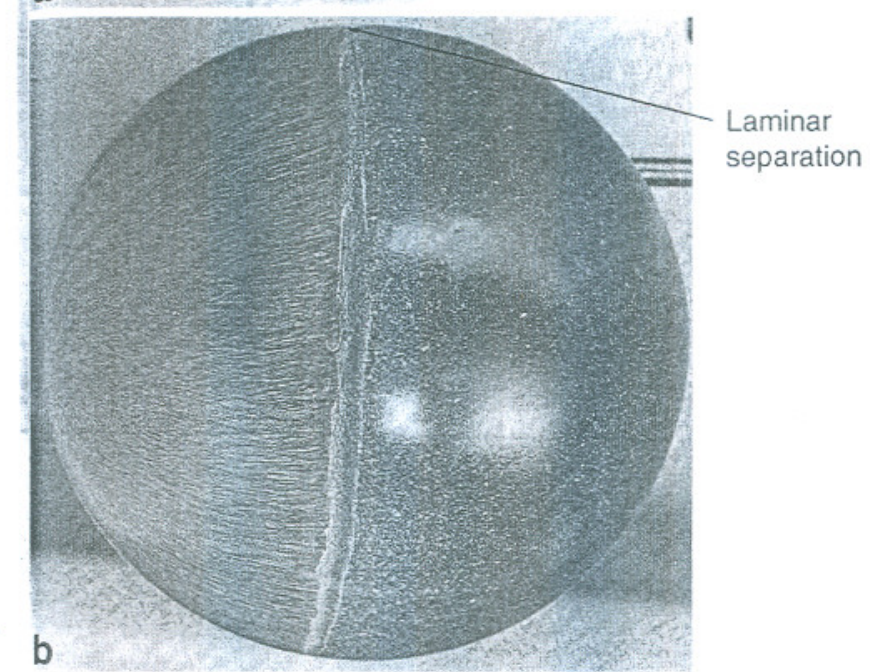

Fig. 9a, b. Effect of ventilation on external flow separation at subcritical Re: a unvented sphere, $R e=6.5 \times 10^{4}$; b vented sphere, $R e=6.5 \times 10^{4}$

al lation on the locations of $\theta_{\mathrm{ls}}, \theta_{\mathrm{br}}$, and $\theta_{\mathrm{ts}}$ are presented in a th composite plot in Fig. 11. Notwithstanding the uncertainties in inferring these locations, there is a weak but definite indication that all the lines move downstream with ventiation, consistent with the pressure measurements reported jy Suryanarayana et al. (1995). The weak upstream travel


Fig. 10a, b. Effect of ventilation on external flow separation at supercritical $R e$ : a unvented sphere, $R e=6.5 \times 10^{5}$; b vented sphere, $R e=6.5 \times 10^{5}$

of $\theta_{\mathrm{br}}$ in the critical regime, as seen for the unvented sphere, is not evident in the presence of ventilation.

\section{4}

\section{Effect of ventilation on vortex shedding characteristics}

Figures 12 and 13 compare the power spectra obtained from water and wind tunnel tests at typical $R e$ in the

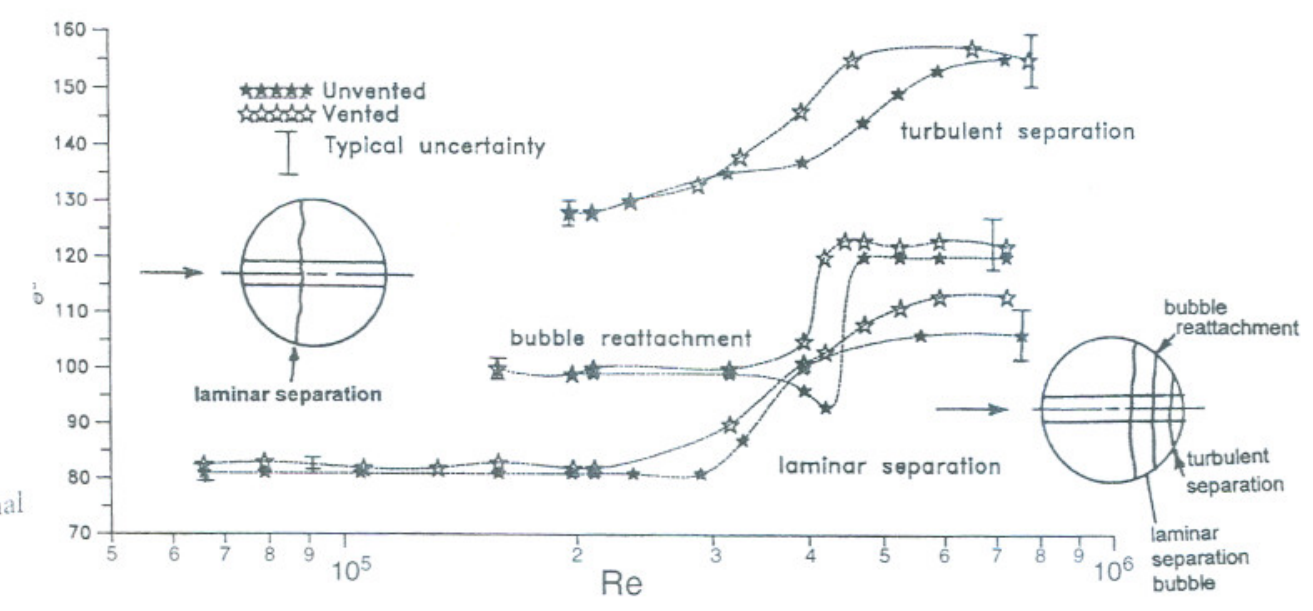

Fig. 11. Effect of ventilation on boundary layer separation and reattachment locations 

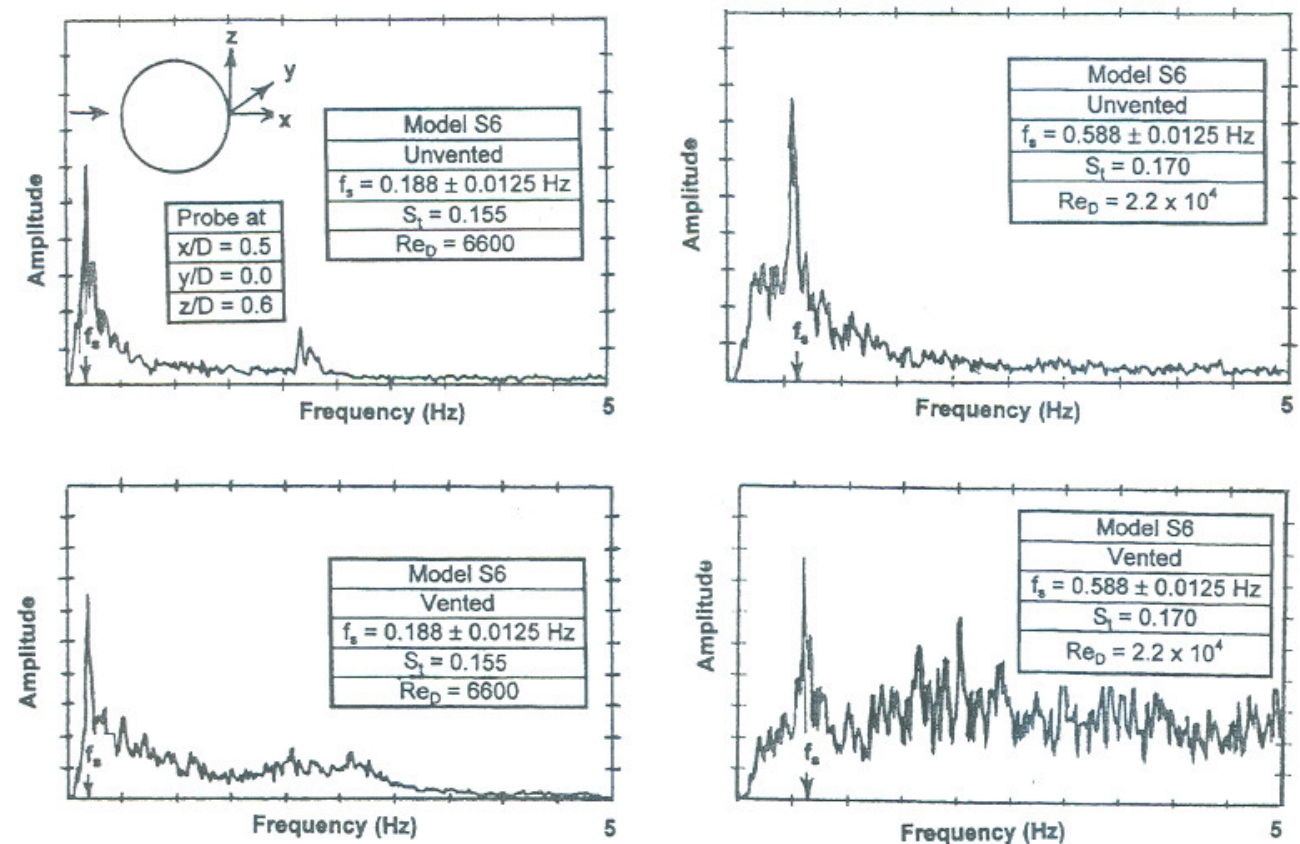

Fig. 12. Effect of ventilation on power spectra of $u^{\prime}$ fluctuations in the near wake at subcritical $R e$ in the water tunnel
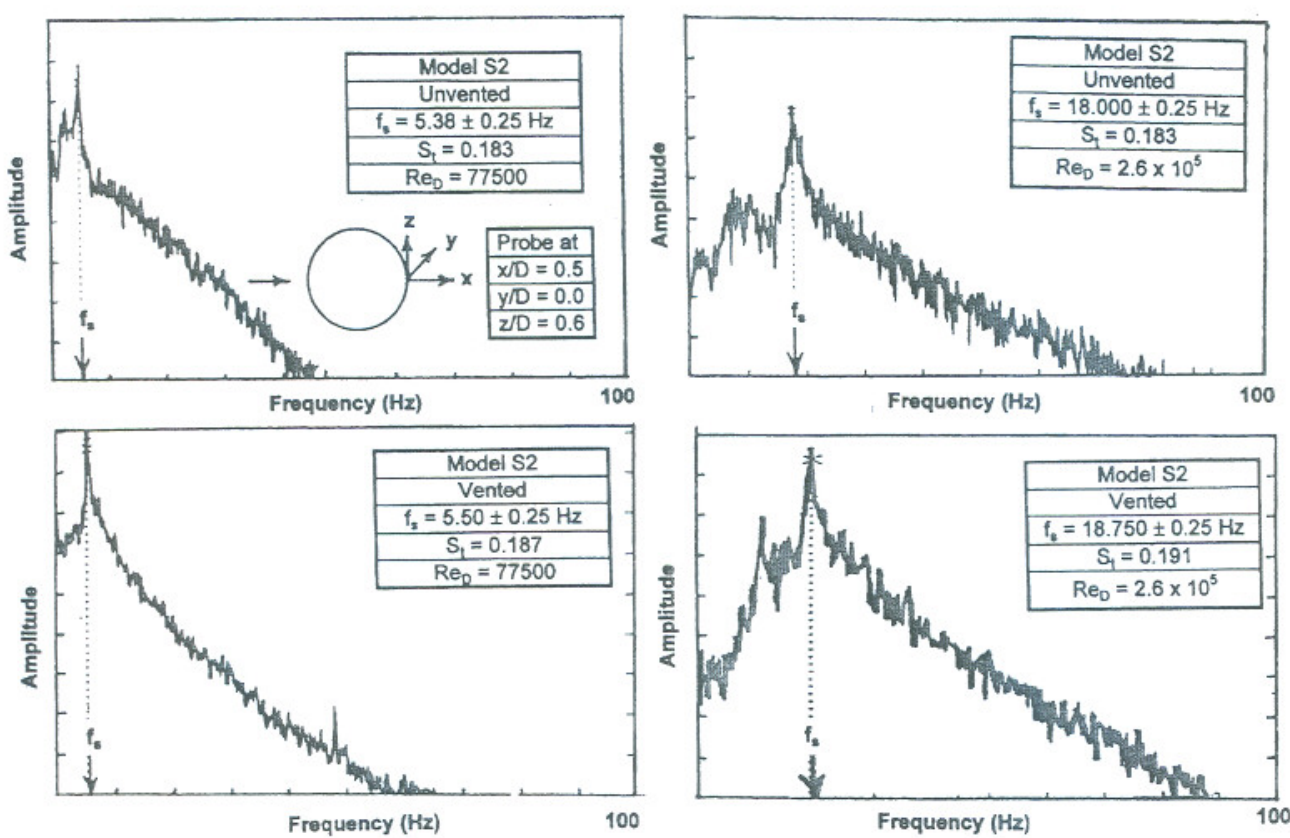

Fig. 13. Effect of ventilation on power spectra of $u^{\prime}$ fluctuations in the near wake at subcritical $R e$ in the wind tunnel

subcritical regime. It is clear that there is only a small change in the vortex shedding frequency in the presence of ventilation. Figure 14 shows the variation in the Strouhal number with $R e$ in the range of $6 \times 10^{3} \leq$ $R e \leq 2.6 \times 10^{5}$.

In the supercritical regime where a significant reduction in total drag (65\%) was observed by Suryanarayana et al. (1993), the power spectral density plots at $R e=4.8 \times 10^{5}$ (Fig. 15) and at $R e=6.5 \times 10^{5}$ (Fig. 16) show a remarkable reduction in the amplitudes at all the frequencies. This feature was observed at several vertical stations within the near wake. The spectra do not show any peak frequency in the presence of ventilation, which is not surprising, since vortex shedding does not exist even in the absence of venting.
It is therefore clear that the observed drag reduction is associated with a significant reduction of wake unsteadiness, presumably caused by the stabilisation/weakening of the randomly rotating 3-D vortical structure of the unvented sphere. The spectra also suggest a more steady boundary layer separation with ventilation. Interestingly, Quadflieg's (1975) experiments involving drag measurements on a sphere show evidence of drag reduction associated with steady separation of the boundary layer and quietening of the near wake. The steady separation was achieved by placing a thin wire close to the location of turbulent boundary layer separation.

Figure 17 shows a comparison of the time-averaged flow pattern of the near wake with and without ventilation, taken from Suryanarayana et al. (1993) and Taneda (1978), 


\begin{tabular}{|c|c|c|c|c|}
\hline \multirow[t]{4}{*}{17} & \multirow[t]{4}{*}{ 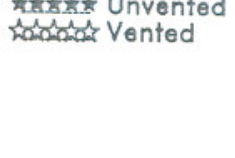 } & Medium & $\begin{array}{l}\text { Water } \\
\text { tunnel }\end{array}$ & \begin{tabular}{|l} 
Wind \\
tunnel \\
\end{tabular} \\
\hline & & Model dia & $79 \phi$ & $200 \phi$ \\
\hline & & Inlet dia & $12 \phi$ & $30 \phi$ \\
\hline & & Exit dia & $12 \phi$ & $30 \phi$ \\
\hline
\end{tabular}

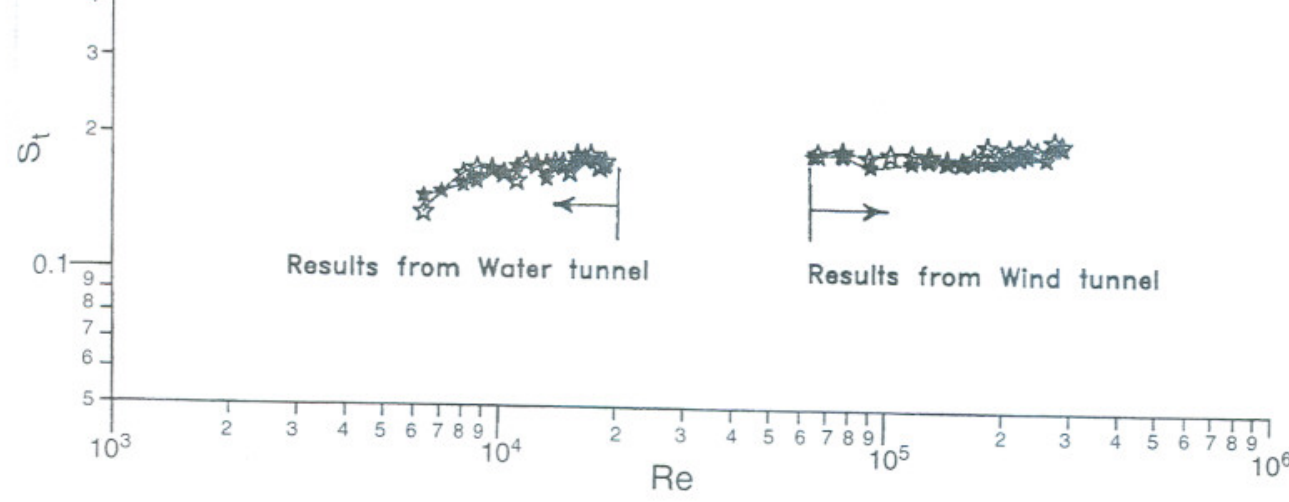

Fig. 14. Effect of ventilation on wake Strouhal number
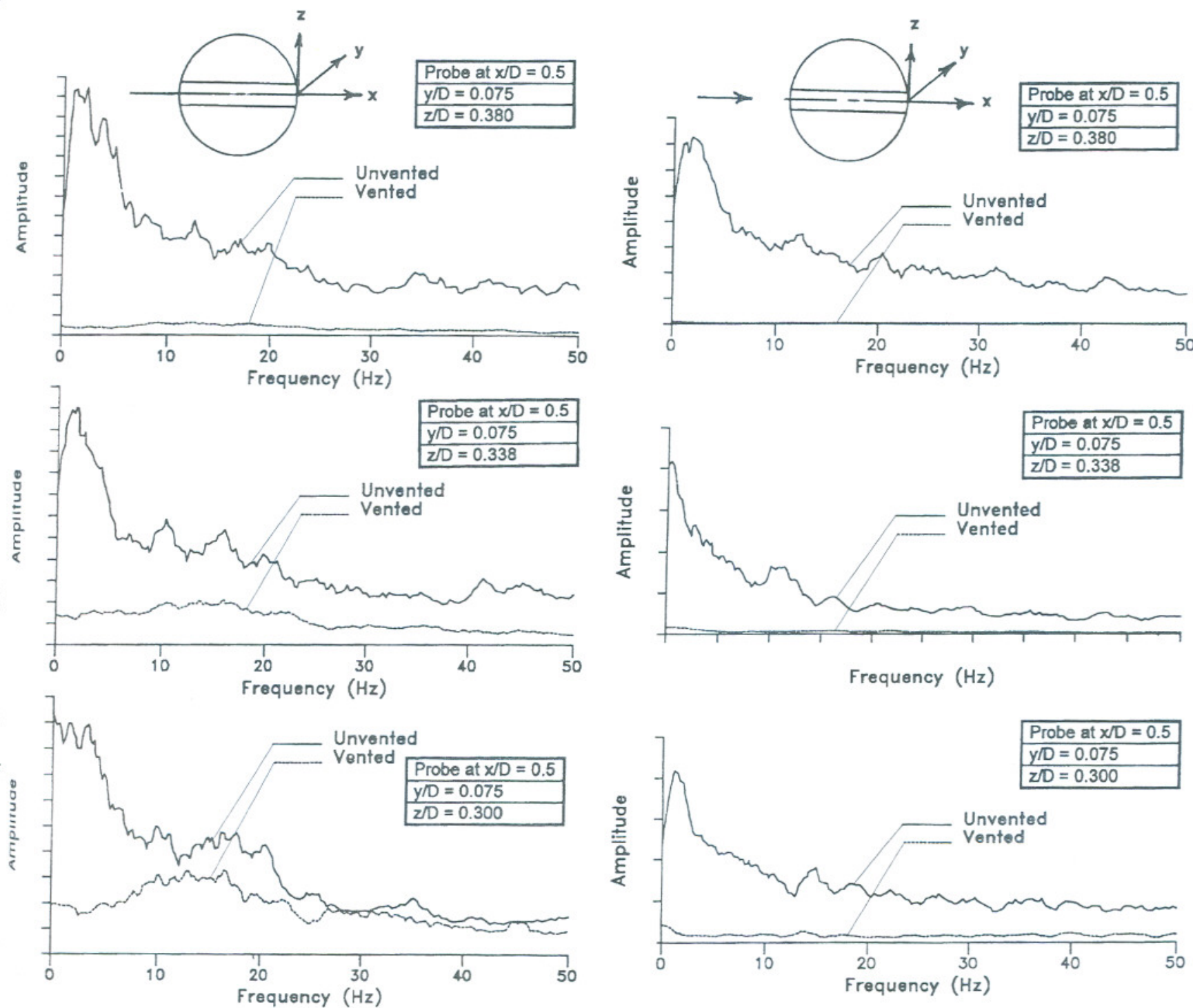

ig. 15. Effect of ventilation on power spectral density of $u^{\prime}$ luctuations in the near wake at supercritical $\operatorname{Re}\left(4.8 \times 10^{5}\right)$

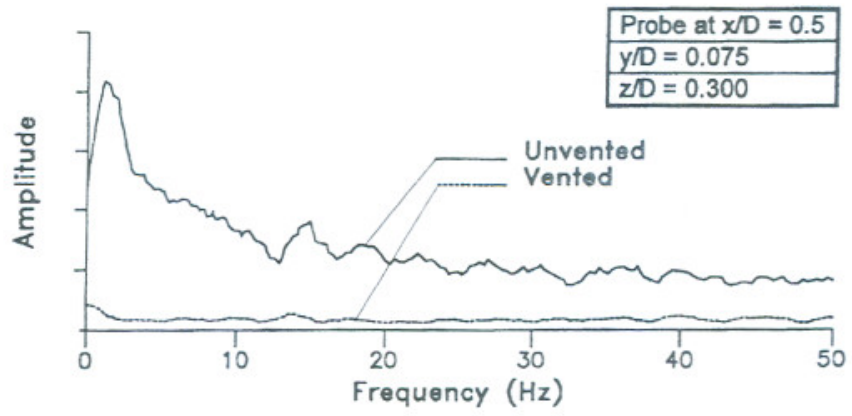

Fig. 16. Effect of ventilation on power spectral density of $u^{\prime}$ fluctuations in the near wake at supercritical $\operatorname{Re}\left(6.5 \times 10^{5}\right)$ 

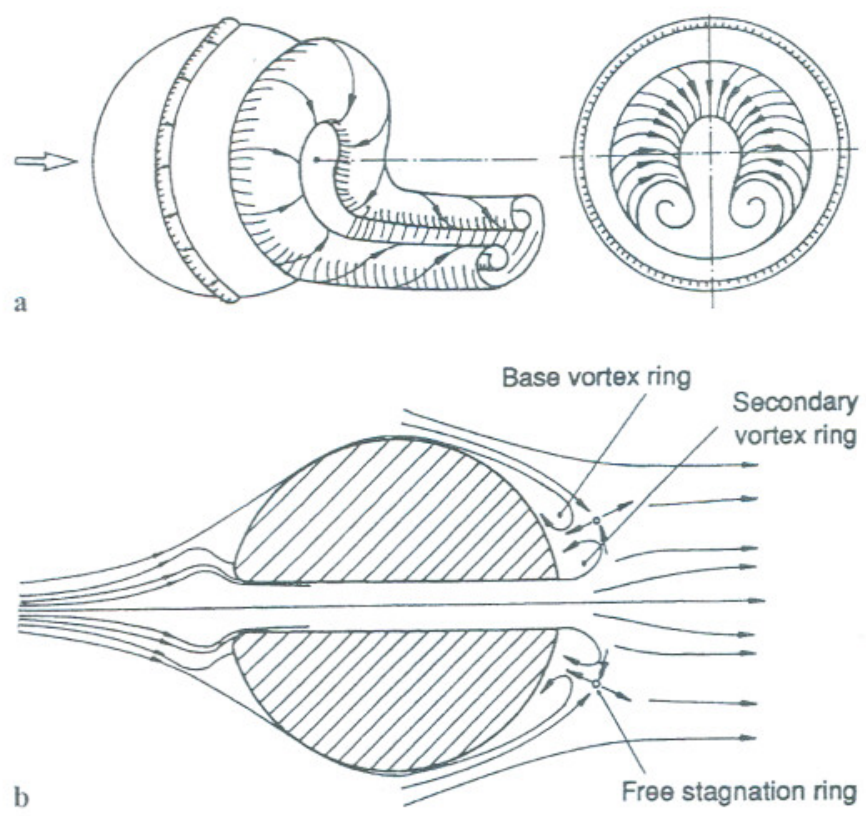

Fig. 17a, b. Effect of ventilation on the near-wake structure of a sphere at supercritical Re: a 3-D vortical structure behind the unvented sphere (Taneda, 1978); b steady-base flow structure behind the vented sphere (Suryanarayana 1993)

respectively. It may be noted that, since the energy required to sustain the randomly rotating vortical structure can be drawn only from the freestream (this reflects as drag), any reduction in wake unsteadiness may be expected to result in drag reduction. The present observation of dramatic reduction in wake unsteadiness due to venting is consistent with the steady base-flow pattern as shown in the figure. A clear correlation between steady wake flow and reduction in drag has been shown by Roshko (1961) for 2-D bluff bodies. The modified structure of the near wake of the vented sphere may be characterized by a pair of counterrotating ring vortices, which have the effect of aerodynamically streamlining the sphere. Thus, ventilation may also be expected to alleviate the random side forces known to exist on spherical objects at high $R e$.
The effects of natural ventilation on the flow past spheres can thus be grouped in two broad categories, viz., weak and strong interaction regimes, as schematically described in Fig. 18. The interaction is weak in the subcritical Re regime, probably because of the early separation $\left(\theta_{1 \mathrm{~s}} \approx 80^{\circ}\right)$ of the boundary layer, which results in a relatively larger distance between the inner and outer shear layers. The basic features of the unvented sphere such as the mean separation characteristics, base pressure, and vortex shedding frequency are therefore largely unaltered, despite the large addition of mass in the near wake. The strong interaction regime is characterized by a delayed separation $\left(\theta_{\mathrm{ls}} \approx 155^{\circ}\right)$, which reduces the distance between the inner and outer shear layers. The boundary layer separation is more steady, resulting in a dramatic reduction in wake unsteadiness, improved base pressure recovery, and notable drag reduction.

\section{4}

\section{Conclusions}

Experiments were carried out to assess the effect of natural ventilation on boundary layer separation and near-wake vortex shedding characteristics of spheres in the Reynolds number ( $R e$ based on diameter) range $6.0 \times 10^{3}$ to $6.5 \times 10^{5}$ in water and wind tunnels. In the subcritical regime of $R e$, ventilation caused a marginal downstream shift in the location of laminar boundary layer separation and a small change in the vortex shedding frequency, consistent with the small change in total drag, as reported by Suryanarayana et al. (1993). In the supercritical Re regime, ventilation caused a downstream shift in the mean locations of laminar boundary layer separation, transitional/turbulent shear layer reattachment and turbulent boundary layer separation; the lines showed significant axisymmetry due to venting at supercritical Re. Hot-wire measurements in the near wake indicate absence of a distinct vortex shedding frequency; the spectra of $u^{\prime}$ signals show a dramatic reduction in the wake unsteadiness at all frequencies across the near wake in the presence of venting. The reduction in wake unsteadiness is consistent with the large reduction in total drag already reported in

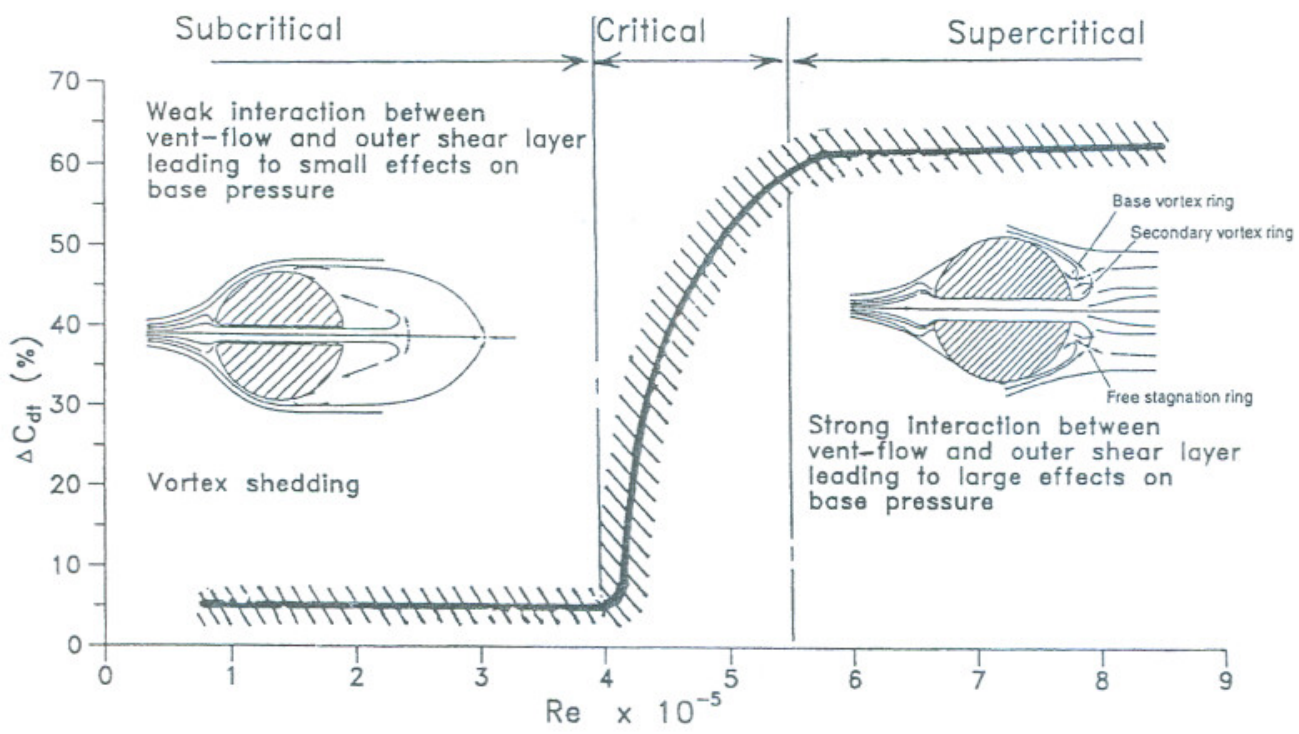

Fig. 18. Effectiveness of natural ventilation in reducing the total drag of a sphere 
Suryanarayana et al. (1993). The effects of natural ventilation can be categorized in two broad regimes, viz., weak and strong interaction regimes. In the weak interaction regime, the broad features of the basic unvented sphere are largely unaltered, despite the large addition of mass in the near wake. Strong interaction is promoted by the closer proximity of the inner and outer shear layers at supercritical Re. This results in a modified/weakened and steady near-wake flow characterized by reduced unsteadiness and small drag.

\section{References}

Achenbach E (1972) Experiments on the flow past spheres at very high Reynolds numbers. J Fluid Mech 54: 565-575

Achenbach E (1974) Vortex shedding from spheres. J Fluid Mech 62 part 2: 209-221

Arakeri VH; Ram G (1984) Visualization of the flow past a sphere near its critical Reynolds number. Curr Sci 53: 180-182

Bearman PW (1967) The effect of base bleed on the flow behind a two-dimensional model with a blunt trailing edge. The Aero. Quarterly XVIII, 207-223

Clift R; Grace JR; Weber ME (1978) Bubbles, drops and particles. Academic Press, London

Cometta C (1957) Vortex shedding from spheres. J Fluid Mech 62 (part 2): 209-221

Fage A (1936) Experiments on a sphere at critical Reynolds numbers. ARC R \& M No. 1766

Igarashi T (1978) Flow characteristics of a circular cylinder with a vent. Bulletin of the JSME 21, 154, 656-664

Kim HJ; Durbin PA (1988) Observations of the frequencies in a sphere wake and of drag increase by acoustic excitation. Phys Fluids 31: 3260-3265
Mair WA; Maull DJ (1971) Bluff bodies and vortex shedding a report on EUROMECH 17. J Fluid Mech 45 (part 2): 209224

Quadflieg H (1975) Verringerung des widerstrandes oberhalb der kritischen Reynolds-zahl bei stationaerer Grenzschichtssabloesung. Abhandlungen aus dem Aerodynamischen Institut der RWTH 22 Aachen, pp 49-51

Raithby GD; Eckert ERG (1968) The effect of support position and turbulence intensity on the flow near the surface of a sphere. Waerme Stoffuebertrag 1: 87-94

Roshko A (1961) Experiments on the flow past a circular cylinder at very high Reynolds numbers. J Fluid Mech 10: 345-356

Sakamoto H; Haniu H (1990) A study on vortex shedding from spheres in a uniform flow. J Fluids Eng 112: 386-392

Suryanarayana GK (1996) On the reduction of drag of a sphere by natural ventilation. Ph.D. Thesis, Department of Aerospace Engineering, Indian Institute of Science, Bangalore, India

Suryanarayana GK; Meier GEA (1995) Effect of ventilation on the flow-field around a sphere. Exp Fluids 19: 78-88

Suryanarayana GK; Pauer H; Meier GEA (1993) Bluff-body drag reduction by passive ventilation. Exp Fluids 16: 73-81

Taneda S (1978) Visual observations of the flow past a sphere at Reynolds numbers between $10^{4}$ and $10^{6}$. J. Fluid Mech 85: 187-192

Viswanath PR (1994) Flow management techniques for base and afterbody drag reduction. PD EA 9405 NAL, Bangalore, India

Wieselberger C; Prandtl L (1914) Mitteilungen aus der Goettinger Modellversuchsanstalt. ZFM 9, 140-145

Zdravkovich MM (1981) Review and classification of various aerodynamic and hydrodynamic means for suppressing vortex shedding. J Wind Eng Ind Aerodyn 7: 145-189 